\title{
La connaissance et le suivi du rendement des réseaux : un outil fondamental pour les distributeurs d'eau
}

\author{
J.L. Demassue \\ Compagnie générale des eaux
}

Depuis longtemps le rendement de réseau est l'outil principal, quelquefois unique, de la mesure de la performance d'un réseau. Il quantifie le pourcentage d'eau de distribution effectivement utilisée par rapport à l'eau nécessaire à l'alimentation du réseau.

Depuis quelque temps l'utilisation conjointe d'autres critères caractéristiques permet d'analyser encore plus précisément le fonctionnement de celui-ci.

D'un côté le distributeur introduit une certaine quantité d'eau en tête de son réseau par un ou plusieurs points d'injection (volume mis en distribution).

De l'autre, les clients à qui est destinée cette eau vont utiliser chacun une partie de ce qui a été introduit dans le réseau.

Entre ces deux extrémités une partie de l'eau introduite est perdue. La connaissance de ces volumes perdus passe par celle de l'ensemble des volumes utilisés d'une façon ou d'une autre.

La première partie de cet exposé est consacrée à la détermination de ces différents volumes.

Une fois ces éléments connus, il faudra déterminer des critères caractéristiques et délimiter les valeurs acceptables ou non de ceux-ci, c'est la deuxième partie de l'exposé.

L'AGHTM, association qui représente l'ensemble des services de distribution d'eau de France, s'est penchée sur cette question. Le groupe de travail constitué à cet effet, dont on $\mathrm{m}$ 'a confié l'animation, s'est attaché à normaliser les différents termes employés (1). L'utilisation rationnelle de ceux-ci devrait permettre de mieux mesurer les performances et faciliter les comparaisons entre différents réseaux.

On ne peut cependant parler de performance d'un système sans évoquer les hommes qui le gèrent.

Dans le cas d'un système complexe comme un réseau d'eau potable, champ d'action de phénomènes subtils enchevêtrés, cette mesure passe avant tout par la mise en place d'hommes qualifiés. Les réseaux (physiques ou commerciaux) ne valent qu'en fonction de la valeur des hommes qui les animent. Conformément à la loi de la «variété requise » (loi d'ASHBY), le degré de complexité du réseau humain régulateur doit être supérieur à celui du réseau régulé (2). Cette règle s'applique aussi aux réseaux de distribution d'eau potable.

Avant de tenter d'en définir les performances, il convient encore de préciser les constituants du réseau de distribution. Pour cela nous reprendrons la définition qu'en donne l'AGHTM (1).

Un réseau de distribution est un ensemble cohérent :

- de réservoirs et d'équipements hydrauliques,

- de conduites de transfert (ou feeders),

- de conduites de distribution,

- de conduites de branchement,

- de points de livraison,

- de tous les appareils de robinetterie et de régulation nécessaires.

Cette précision est importante notamment pour la partie "critères caractéristiques". En effet, dans l'utilisation de certains d'entre eux concernant les conduites, il est rarement précisé s'il s'agit de conduites de transfert, de distribution ou de branchement, ce qui peut induire des différences très importantes et donc des erreurs d'appréciation.

\section{Mesures et estimations}

Nous l'avons vu, la mesure de la performance d'un réseau consiste surtout à connaitre le volume d'eau perdu. Encore faut-il définir le terme "perdu ». Nous considérerons qu'il s'agit du volume d'eau qui n'aura servi à personne, introduit dans le réseau en pure perte, uniquement pour maintenir une qualité de distribution constante ; pour cela nous commencerons par définir le volume qui aura été utilisé d'une façon ou d'une autre.

\subsection{Connaissance du volume utilisé}

Nous reprendrons les différentes catégories de volumes définies par l'AGHTM (1).

\section{Network efficiency knowledge and monotoring : an important tool for water suppliers}

Network efficiency has been for a long time a tool for measuring the network performance. It is possible to analyse with more accuracy its working with other criteria.

The use of these criteria makes necessary the definition of the different kinds of water volumes that deal with network management. 


\subsubsection{Volume comptabilisé}

\section{Définition :}

Le « volume comptabilisé » résulte des relevés des appareils de comptage des abonnés.

Le terme «comptabilisé » est à prendre dans le sens où il comptabilise les relevés de l'ensemble des compteurs des abonnés à l'exclusion des compteurs d'exportation. C'est souvent, ou cela devrait être, la part la plus importante du volume utilisé. Il peut différer du volume facturé, notamment en cas d'application de forfaits de facturation (n'existent pratiquement plus en France) ou de dégrèvements.

L'utilisation de ce volume dans la mesure de performance doit s'accompagner de précautions, car généralement ce volume est le résultat du relevé de très nombreux compteurs effectué sur une période relativement longue. De ce fait il convient de bien définir la période à laquelle il se rapporte.

La solution idéale serait de pouvoir relever l'ensemble des compteurs des abonnés sur une seule journée, afin de pouvoir comparer valablement le volume correspondant avec le volume mis en distribution dont le nombre moins important de compteurs permet de les relever facilement en moins d'une journée.

Pour tendre vers le relevé « instantané » des compteurs des abonnés, plusieurs voies sont explorées. Parmi celles-ci figurent les différentes catégories de report d'index ou de radio relevés. On peut aussi citer une expérience originale (en France) d'autorelevés qui se déroule dans le Pays de Montbéliard depuis 1983 sur 27000 abonnés et qui donne toute satisfaction (3).

\subsubsection{Volume consommateurs sans comptage}

\section{Définition :}

Le « volume consommateurs sans comptage » désigne le volume utilisé sans comptage par des usagers connus, avec autorisation.

C'est le volume utilisé par des usagers, autres que le service d'eau, autorisés pour diverses raisons à utiliser de l'eau sans passer par un appareil de comptage. Il s'agit surtout de besoins publics (lavage des rues, principalement). Il est évalué.

L'évaluation de cette catégorie de volumes peut varier de façon très importante d'un réseau à un autre en fonction notamment du nombre de points de puisage non équipés de système de comptage. Il convient de prendre d'autant plus de précautions que son importance relative est grande.

\subsubsection{Volume de service du réseau}

\section{Définition :}

Le « volume de service du réseau » est le volume utilisé pour l'exploitation du réseau de distribution d'eau.

Il s'agit de l'eau utilisée en toute connaissance de cause par l'exploitant pour le nettoyage des réservoirs ou des purges des conduites par exemple.

Ce volume est lui aussi évalué.

\subsubsection{Volume détourné}

\section{Définition :}

Le «volume détourné » est le volume utilisé frauduleusement.
C'est le volume utilisé par les branchements clandestins, les piquages avant compteurs, la falsification de ceux-ci, l'utilisation illégale des poteaux d'incendie...

Il s'agit aussi d'un volume évalué mais dont l'estimation est encore plus malaisée que les précédentes car elle se rapporte à des événements normalement inconnus.

Seule la bonne connaissance du terrain et du comportement des usagers permettra d'évaluer correctement ce volume.

Même si celui-ci constitue une perte pour l'exploitant il reste cependant un volume utilisé. Heureusement, dans de nombreux pays, il est suffisamment faible pour pouvoir être considéré comme négligeable.

\subsubsection{Volume résultant des défauts de comptage (chez les abonnés)}

\section{Définition :}

Le volume « défaut de comptage » est le volume résultant de l'imprécision et du dysfonctionnement des organes de comptage, des oublis des relevés, des erreurs d'évaluation et de lecture.

Il représente la différence entre le volume réellement utilisé par l'ensemble des utilisateurs disposant d'appareils de comptage et le volume comptabilisé. Cette différence résulte de mauvaises évaluations (lorsqu'il y en a), d'erreurs de relevé d'index, mais surtout de l'inexactitude des compteurs des abonnés.

$\mathrm{Si}$ les deux premières causes peuvent occasionnellement surévaluer le volume réellement utilisé, la troisième tend généralement à le sous-évaluer.

Trois raisons sont à l'origine de l'inexactitude des compteurs :

a. par conception un compteur n'est rigoureusement « exact " qu'à un seul débit. Or la plage d'utilisation d'un branchement, et donc du compteur, est d'une part très large (de la micro-fuite à la capacité maximale du branchement) et d'autre part semble très souvent répartie de façon aléatoire.

L'optimisation du comptage dépendra bien sûr du choix du compteur et notamment de son type mais aussi et surtout de son calibrage qui est une démarche essentielle dans la mesure du volume comptabilisé.

Il semble que, lorsque ce calibrage n'a pas été étudié correctement, les habitudes tendent à surdimensionner les compteurs (par exemple faire correspondre le diamètre du compteur à celui du branchement). Cette situation se traduit par une utilisation importante du compteur dans sa plage de sous-comptage, et donc par une sous-évaluation du volume comptabilisé.

En France, les distributeurs utilisent des tableaux de correspondance donnant pour chaque type de compteur, le diamètre en fonction du nombre de logements alimentés. Ces valeurs sont à considérer comme des ordres de grandeur.

b. par vieillissement. A part certains types de compteurs de vitesse, les compteurs ont en général tendance à souscompter en vieillissant.

Le suivi du parc de compteurs et le remplacement de ceux-ci en temps voulu influencera de façon significative la mesure du volume comptabilisé.

S'il est convenu qu'un compteur n'est pas éternel, son remplacement prématuré induit des dépenses inutiles. 
Théoriquement, le remplacement devrait intervenir lorsque l'erreur de comptage dépasse un certain seuil déterminé dans le règlement des eaux (c'est la limite du préjudice acceptable pour l'usager ou pour le service).

La conjugaison de ces différents éléments peut entraîner un sous-comptage pouvant aller jusqu'à 7 ou $8 \%$ du volume comptabilisé, peut-être plus dans certains cas. Seuls des spécialistes du comptage ayant une grande expérience du problème pourront donner un avis global en fonction de l'âge du parc, du type des compteurs, de la plus ou moins bonne adéquation des calibres, de la qualité de l'eau, etc...

c. Par mauvais choix. Le choix du type et de la classe des compteurs à mettre en place peut avoir une incidence importante sur la précision du volume comptabilisé. C'est le cas, par exemple, pour des compteurs de type volumétrique utilisés avec une eau véhiculant des particules abrasives très fines qui vont rayer très rapidement la bâche des compteurs et accentuer ainsi le phénomène de souscomptage.

Nota: Toutes les remarques relatives aux défauts de comptage s'appliquent bien évidemment aux compteurs de mise en distribution (en tête de réseau) avec plus encore de conséquences compte tenu du volume mesuré et du nombre peu important de ceux-ci. Une mauvaise adéquation des compteurs (ou systèmes de comptage) de mise en distribution peut entraîner des erreurs très importantes, généralement en sous-comptage pouvant aboutir quelquefois à des rendements aberrants.

\subsection{Volume perdu non utilisé}

Nous venons de voir l'ensemble des volumes utilisés. La différence entre le volume mis en distribution et les volumes utilisés constitue ce que nous appellerons le « volume perdu non utilisé ».

Celui-ci se décompose en deux catégories, le volume gaspillé et le volume des fuites.

\subsubsection{Volume gaspillé}

\section{Définition :}

Le volume gaspillé est le volume perdu en raison d'incidents d'exploitation.

Ce volume est à mettre au compte de l'exploitant du réseau, comme celui de service du réseau. Il en diffère cependant parce qu'il est involontaire. Il s'agit surtout du débordement des réservoirs ou de vidanges mal fermées. Ce volume revêt un caractère exceptionnel. Il est évalué. Cette perte n'est pas significative de l'état d'un réseau. Elle peut être importante pendant une période courte. Lorsqu'elle prend des proportions excessives, elle devient significative du fonctionnement d'un service.

\subsubsection{Volume de fuites}

\section{Définition :}

Le volume de fuites est le volume résultant des défauts d'étanchéité du réseau.

Cette notion concerne tous les ouvrages du réseau: réservoirs (à ne pas confondre avec le débordement), conduites de transfert, de distribution et de branchement, appareils de robinetterie, de régulation...
Les plus connues, et certainement les plus nombreuses, sont les fuites sur conduites (de transfert mais surtout de distribution et de branchements).

Nous venons de passer en revue l'ensemble des catégories de volumes constituant le volume mis en distribution.

Même si cet exercice paraît fastidieux il est indispensable à la connaissance du fonctionnement d'un réseau et en conséquence à la mesure de la performance de celui-ci.

Nous avons vu qu'il était possible moyennant certaines précautions de connaître la valeur approchée du volume utilisé, somme algébrique des volumes comptabilisés, consommateurs sans comptage, service du réseau, détourné et défaut de comptage.

Ce volume regroupe toutes les utilisations, maitrisées ou non, de l'eau du réseau. Il est de même intéressant de définir l'ensemble des volumes qui sont perdus pour l'exploitant, nous l'appellerons LE VOLUME DES PERTES EN DISTRIBUTION. Il représente la somme algébrique des volumes de fuites, gaspillés, détournés et défaut de comptage.

La caractéristique commune de ces pertes est qu'elles ne sont pas mesurables.

Des techniques de mesures et de diagnostics permettent, en prenant certaines précautions, d'appréhender ces valeurs de façon satisfaisante.

\section{Critères de mesure de la performance des réseaux}

Après avoir mesuré et estimé tous ces volumes, préambule obligatoire, il est intéressant de définir un certain nombre de critères qui vont nous permettre de mesurer l'efficacité de la gestion et de contrôler par leur dérive dans le temps, l'évolution de cet équipement essentiel qu'est le réseau de distribution.

Le premier de ces critères dans le cas d'un réseau est souvent le rendement. C'est un outil bien connu pour mesurer la performance d'un système physique. Il est censé caractériser les pertes. Pour un réseau il quantifie le pourcentage d'eau de distribution utilisée d'une manière ou d'une autre par rapport à l'eau nécessaire à l'alimentation du réseau.

Derrière ce concept général beaucoup d'ambiguités et de variantes existent.

Si le rendement est simple d'utilisation, encore faut-il savoir exactement ce que représentent le numérateur et le dénominateur du ratio qui le caractérise.

A partir des différentes catégories de volumes définies précédemment nous allons définir plusieurs types de rendements.

\subsection{Rendement primaire RI (exprimé en \%)}

$$
R 1=100 \cdot \frac{\mathrm{V} \text { comptabilisé }}{\mathrm{V} \text { mis en distribution }} .
$$

C'est le rendement le plus simple à calculer car, pour les services dont les abonnés sont équipés de compteur (c'est le cas de la France notamment), le numérateur et le dénominateur sont connus chaque année.

Il faut cependant rappeler, et cela est valable pour l'ensemble des rendements, que pour que ce chiffre ait une valeur mathématique, il est impératif que les volumes correspondent à la même période. 
Nous l'avons évoqué dans les commentaires sur le volume comptabilisé, mais ce volume, pour les services importants, résulte souvent de périodes de relevés étalées sur plusieurs semaines. Il convient donc de prêter une attention toute particulière à la publication de ce chiffre en essayant de limiter au maximum la durée des relevés et en vérifiant que la période moyenne entre deux relevés est constante. Elle doit correspondre à celle des volumes mis en distribution.

Le rendement primaire ne permet pas de comparer deux réseaux de configurations différentes. Le nombre (ainsi que l'absence ou l'existence de comptage) des bouches d'arrosage et de lavage par exemple peut générer des différences importantes de rendement primaire entre deux services apparemment identiques.

En première analyse, pour un réseau donné, l'évolution de ce rendement sur plusieurs années importe plus que sa valeur absolue.

\subsection{Rendement consommateurs}

C'est le rendement qui prend en compte tous les volumes utilisés par les consommateurs, équipés ou non d'un compteur, on peut parler du volume «clients».

$$
R 2=R 1+P 1
$$

$P 1$ : pourcentage consommateurs sans comptage.

$$
P 1=100 \cdot \frac{\text { V consommateurs sans comptage }}{\mathrm{V} \text { mis en distribution }} .
$$

Le numérateur de $P 1$ doit être analysé avec précaution car parmi les volumes estimés, il est généralement le plus important (à l'exception du volume de fuites). Il peut être très différent d'un service à un autre en fonction du nombre des appareils installés et du nombre de ceux équipés d'un compteur.

Dans l'expression de $R 2$ il est souhaitable de faire état de $R 1$ et $P 1$ plutôt que de $R 2$ seul. En effet pour deux résultats $R 2$ identiques, la formulation des $R 1$ et $P 1$ permet une meilleure approche de la connaissance de ces réseaux.

Cette remarque s'applique de même pour le rendement suivant.

\subsection{Rendement net}

Il est défini comme : $R 3=R 1+P 1+P 2$

$P 2=$ pourcentage service du réseau.

$$
P 2=100 \cdot \frac{\mathrm{V} \text { service du réseau }}{\mathrm{V} \text { mis en distribution }} .
$$

Ce rendement est très intéressant puisqu'il compare la totalité de l'eau utilisée sciemment à la quantité nécessaire à l'alimentation permanente du réseau. Il traduit nettement la notion de perte d'eau.

Il faut cependant noter le risque d'utilisation de ce rendement qui pourrait inciter l'exploitant à utiliser une quantité d'eau abusive pour ses propres besoins (volume de service du réseau) : en effet à consommation et utilisation constantes, l'augmentation du volume de service du réseau fait augmenter parallèlement le rendement net.

C'est pourquoi comme nous le signalions précédemment il est souhaitable de faire référence à $R 1+P 1+P 2$ sous forme d'une suite de trois chiffres plutôt qu'à $R 3$ seul.
Cette solution permet de considérer $P 2$ dont l'importance est quelquefois justifiée (dans certains services ruraux, par exemple).

D'autres rendements peuvent évidemment être calculés mais il faut signaler que l'utilisation unique d'un ou plusieurs rendements, même si elle est intéressante, est notablement insuffisante pour la mesure de la performance d'un réseau. Elle peut même conduire à des interprétations erronées. En effet elle ne fait intervenir en aucune façon la longueur du réseau par exemple et privilégie les services à forte densité urbaine et à consommations importantes.

C'est pourquoi il est impératif de disposer d'autres indices, notamment les indices linéaires de fuites, de perte, de consommation ou encore de réparations. Utilisés depuis longtemps par de nombreux services en France dont ceux de la Compagnie Générale des Eaux (4), ils permettent de mieux connaître les véritables performances du réseau, leur évolution dans le temps et de fixer des objectifs à atteindre.

\subsection{Indice linéaire de fuites}

$I L F=\frac{\mathrm{V} \text { de fuites }}{\mathrm{L} \text { conduites (transfert }+ \text { distribution }+ \text { branchements) }}$ exprimé en $\mathrm{m}^{3} / \mathrm{j} . \mathrm{km}$.

Cet indice permet d'appréhender l'état d'étanchéité du réseau. Contrairement aux différents rendements, il n'est pas influencé par l'utilisation de l'eau par les différents usagers. Il permet de comparer l'état physique de deux réseaux. C'est un outil de gestion indispensable, à condition bien sûr de prendre toutes les précautions nécessaires au calcul du volume de fuites. Il permet facilement de définir des valeurs limites en fonction du type de réseau que l'on a à étudier, ce qui est beaucoup plus difficile avec la simple utilisation du rendement.

De façon générale, plus un réseau fournit d'eau au mètre de conduite, plus le diamètre moyen est important.

Le volume des fuites, et donc l'indice linéaire de fuite, est normalement fonction du diamètre moyen des conduites du réseau. Comme cette notion n'est pas facile à appréhender, il est fait appel à une autre qui lui est logiquement liée, l'indice linéaire de consommations défini ci-après.

\subsection{Indice linéaire de consommation net}

\section{$I L C N=$}

$\mathrm{V}$ (comptabilisé + consom. sans comptage + service du réseau)

$\mathrm{L}$ conduites (transfert + distribution + branchements)

$$
\mathrm{m}^{3} / \text { jour.km . }
$$

Il donne une notion d'utilisation du réseau.

Cet indice, à lui seul, ne permet pas d'apprécier les performances d'un réseau. En revanche il permet de classer les réseaux en fonction de la densité du volume délivré.

Ainsi on peut classer un réseau en rural lorsque son $I L C N$ est compris entre 0 et $10 \mathrm{~m}^{3} / \mathrm{j} . \mathrm{km}$.

Un service urbain ou industriel dépasse $30 \mathrm{~m}^{3} / \mathrm{j} . \mathrm{km}$. Un service entre les deux sera considéré comme semi-rural ou semi-urbain.

Dans l'absolu, l'exploitant aura intérêt à réduire le plus possible les volumes perdus en fuites. L'expérience et la logique montrent que plus le réseau est étanche plus il est difficile et onéreux de faire baisser ce volume de fuites. 
Il existe un équilibre économique entre le coût des recherches et réparations et le coût des pertes. Celui-ci est fonction de l'importance du réseau, donc de l'indice de consommation net.

Si donc on veut apprécier les performances d'un réseau il convient d'observer l'indice linéaire de fuites en fonction de l'indice linéaire de consommation.

Des années d'observation de ces critères sur des réseaux de toutes tailles et de tous types ont permis à la Compagnie Générale des Eaux de se fixer des seuils en deçà desquels on peut considérer que toute nouvelle campagne de recherche d'amélioration coûtera plus cher que l'économie envisagée.

Ces observations aboutissent aux recommandations résumées dans le tableau suivant:

\begin{tabular}{|c|c|c|c|}
\hline $\begin{array}{l}\text { Indice linéaire de consom- } \\
\text { mation net en } \mathrm{m}^{3} / \mathrm{j} . \mathrm{km}\end{array}$ & 0 à 10 & 10 à 30 & $>30$ \\
\hline Catégorie du réseau & rural & semi-rural & urbain \\
\hline $\begin{array}{l}\text { Indice linéaire de fuites } \\
\text { acceptable en } \mathrm{m}^{3} / \mathrm{j} . \mathrm{km}\end{array}$ & $<2$ & $<5$ & $<10$ \\
\hline
\end{tabular}

Ces valeurs sont à considérer en tant qu'ordre de grandeur et en première approche. Le jugement devra être corrigé en fonction notamment de l'importance et de la nature de la ressource disponible, de l'âge du réseau, de sa structure, de la pression moyenne de distribution, etc...

\subsection{Indice linéaire de réparations}

Cet indice très complémentaire de l'indice de fuites donne une excellente indication de la difficulté qu'il y a à atteindre et maintenir un objectif de rendement.

La comparaison de ces indices pour des services ayant des $I L F$ équivalents permettra notamment d'avoir une idée de l'état général du réseau.

Le fait de scinder cet indice en un « indice de réparation branchements » et un « indice de réparation conduites principales (transfert et distribution)" permettra de juger encore plus précisément de l'état de chaque type de conduites et notamment de diriger une politique de renouvellement.

L'utilisation de cet ou de ces indices par secteurs de distribution permettra de même d'orienter les opérations d'ordre curatif et préventif.

Nota : la notion de réparation doit être prise dans le sens de réparation de conduites ou d'appareils présentant des fuites significatives, elle doit exclure par exemple les changements de joints de compteurs.

D'autres indices peuvent aussi présenter quelque intérêt, par exemple L'INDICE LINÉAIRE DE PERTES EN
DISTRIBUTION qui fait apparaître au numérateur le volume des pertes en distribution, soit la somme algébrique des volumes de fuites, gaspillé, détourné et défaut de comptage.

Ou encore L'INDICE SURFACIQUE DES PERTES qui au volume des pertes au numérateur associe au dénominateur la surface intérieure de l'ensemble des conduites du service.

Exprimé en $\mathrm{m}^{3} / \mathrm{m}^{2}$ il permet d'une certaine façon de prendre en compte la taille des conduites.

On peut de même citer L'INDICE DÉMOGRAPHIQUE DE CONSOMMATION NET ou au même numérateur que précédemment est associée la population recensée au dénominateur. Cet indice permet de juger de l'utilisation moyenne par habitant ou «équivalent habitant " en fonction de la concentration.

\section{Conclusion}

La mesure de la performance d'un réseau est une démarche compliquée qui, si elle veut être crédible, passe par une organisation rigoureuse et un contrôle de cohérence permanent de tous les chiffres utilisés.

En plus du contrôle fréquent des différents organes de comptage, dont la nécessité est encore plus importante pour la mise en distribution, il convient de raisonner sur des « ensembles de taille acceptable».

Derrière cette notion très vague se cache toute l'expérience et les capacités d'analyse que doivent posséder les responsables de réseaux pour structurer ceux-ci en secteurs dont la taille dépendra des spécificités locales mais qui, surtout, évitera le phénomène de lissage propre aux gros ensembles. Chacun de ces secteurs sera équipé d'un comptage de mise en distribution et chaque client sera affecté au secteur correspondant.

L'ensemble de ces démarches devra être confié à du personnel qualifié. Si l'on connaît de par le monde un certain nombre de spécialistes d'usines de traitement il est surprenant de ne pas trouver la même situation pour les réseaux d'eau. Comme nous le citions en préambule, le réseau est un système complexe, champ d'action de phénomènes subtils enchevêtrés. Sa gestion doit être l'affaire de spécialistes reconnus comme tels.

\section{Bibliographie}

[1] AGHTM. - «Rendement des réseaux d'eau potable. Définition des termes utilisés ». Techniques, Sciences, Méthodes $\mathrm{n}^{\circ} 4$ bis 90 .

[2] P.L. GIRARDOT. - "Chaînes et trames: le tissu des réseaux». Annales des Mines, avril 1991.

[3] B. Buchholzer, J.L. Demassue. - "Autorelevé des compteurs d'eau potable du District Urbain du Pays de Montbéliard . Techniques, Sciences, Méthodes n³-88.

[4] J. BuSTARRET. - * Mesure et signification des rendements de réseau. Moyens et méthodes pour rechercher les fuites d'eau ". Techniques, Sciences, Méthodes n¹1-78. 